\section{How to stop blogging}

\author{
Organizers have only two options for their \\ meetings: open or closed.
}

$s$ the scientific conference in its death throes? Researchers have long anguished about the hyper-competitive culture that leads attendees to suppress their most interesting unpublished results. Such protectiveness can only be worsened by the increasing dissemination of results beyond the conference hall by bloggers.

Those who attempt to regain control of communications face outcry. Certain corners of the Internet have been erupting in argument in the past weeks following an announcement by Cold Spring Harbor Laboratory in New York that it will henceforth require scientists who blog to ask the permission of presenters before firing up computers or mobile phones and publicizing their findings.

We are in the midst of a clash of conference-going cultures (see Nature 459, 1050-1051;2009). Attendees who have taken to blogs and other social-media applications such as Twitter and Friend Feed will value the instantaneous communication of fact, conjecture and commentary as a way to network beyond badge-holders. Most researchers, in contrast, will focus on the science and ways to network with fellow attendees. If they are aware of social-networking applications, they are likely to regard them as distractions at best. At worst, they will fear them as tools to undermine and scoop, to release data not ready for consumption by anyone other than the trusted colleagues who bothered to make it to their talk or walk up to their poster and start asking questions.

Conference organizers are stuck in the middle. They want to let the world know that their meetings are worthwhile, and yet they also want to attract speakers presenting the newest and most cuttingedge findings. So how to protect speakers from having sensitive, unfinished or 'scoopable' work broadcast to the world?

\section{Adieu to nuclear recycling}

President Barack Obama should be applauded for his decision to scrap commercial reprocessing.

T his week, US President Barack Obama has been grabbing headlines with his efforts to revitalize the Strategic Arms Reduction Treaty - a US/Russian agreement to reduce the nuclear arsenals of both nations.

Such efforts will be applauded worldwide, but another decision by the Obama administration deserves equal acclaim. On 29 June, the president quietly cancelled a lengthy environmental review that was the first step in allowing the resumption of commercial nuclear reprocessing in the United States. Nuclear reprocessing chemically separates uranium and plutonium from spent nuclear fuel so that it can be reused in specialized reactors. The same technique can be used to purify material for nuclear weapons, and it is partly for that reason that the United States decided to halt reprocessing in the 1970s.

Obama's predecessor, George W. Bush, sought to reverse that
Cold Spring Harbor's strategy is to unilaterally require all bloggers to ask permission: an opt-in strategy for presenters. Others have proposed an opt-out strategy: speakers can affix a logo to their slides or posters that says, in effect, "These results are off limits, for now."

Neither of theses solutions is sustainable, however. Anyone who's heard the chime of a digital camera starting up in the middle of a session knows that clear, sometimes quite threatening, restrictions on photography are regularly ignored. So too it will be with social media users, for whom jotting down their personal reactions to a talk or poster on the fly is simply second nature.

So, what is the answer? Nature has made the case that blogging by researchers is good. Critical discussion of worthy results should not in principle be restricted to walls of a conference hall or even the pages of a journal. Any meeting to which anyone can register is fair game for all available "Anyone who's heard the chime of a digital camera in the middle of a session knows that restrictions are regularly ignored." communications technologies - and any rules that cannot be policed will be ignored anyway.

But some of the most valuable scientific meetings are more focused and deliberative, and are by invitation only. These intellectually intimate gatherings are valuable precisely because the researchers who attend feel free to speculate and to stimulate their colleagues, and to try to establish new research agendas. Whether an attendee is a blogger or a reporter or a practising researcher is immaterial. The meeting is 'off the record', and all have to sign up to that.

Closed or open? Meeting organizers need to be clear in their minds which of these two approaches is appropriate, and be explicit about it from start to finish. The consequence that, in competitive fields, presentations at open meetings will become even more protective and boring is an inevitable consequence of the Internet.

decision. He thought that reprocessing could be part of a broader approach that would see used fuel from non-nuclear-weapons states brought to the United States for reprocessing. As part of the Global Nuclear Energy Partnership programme, Bush advocated the construction of a demonstration commercial reprocessing plant, and an environmental review was already under way when Obama came into office.

Such a plant, had the plans been allowed to continue, would have been both costly and counterproductive. Proliferation worries aside, reprocessing is complex, expensive and creates a liquefied stream of highly radioactive waste that is difficult to dispose of. The technology is likely to be needed within the next two decades, so Obama is right in his decision to allow research into ways to improve reprocessing, while constraining the programme to one of basic science.

The decision to halt commercial nuclear recycling sends a clear message that the United States is committed to nuclear non-proliferation. Such decisions, together with diplomacy such as that taking place in Russia, are deliberate and encouraging first steps towards building an international consensus on reducing the threat from nuclear weapons. 